\title{
Ion Beam Induced Charge Studies of CdZnTe Grown by Modified Vertical Bridgman Method
}

\author{
M. C. Veale, P. J. Sellin, J. Parkin, A. Lohstroh, A. W. Davies, and P. Seller
}

\begin{abstract}
Ion Beam Induced Charge (IBIC) and time resolved digital IBIC techniques have been used to map the electronic properties of CdZnTe manufactured by Yinnel Tech Inc. The $2 \mathrm{MeV}$ proton scanning microbeam at the University of Surrey Ion Beam Centre was used to map the charge transport properties of both holes and electrons at room temperature. The electron response of the detector showed good uniformity whereas the hole response showed significant variations across the bulk.
\end{abstract}

Index Terms-CdZnTe, charge transport properties, digital pulse processing, IBIC, trapping.

\section{INTRODUCTION}

$\mathbf{F}$ OR many years CdZnTe has been a leading candidate for use as a room temperature semiconductor radiation detector. Large scale implementation of these detectors has been hampered by the difficulties of producing large quantities of homogeneous material. Impurities introduced during the growth process form defects in the material resulting in charge carrier trapping that limits the charge collection efficiency (CCE). In the case of holes this problem is particularly severe.

In this paper the variation in the room temperature charge transport properties of a spectroscopic grade $\mathrm{CdZnTe}$ detector have been studied on a micrometer scale. The crystals were grown by Yinnel Tech Inc via the Modified Vertical Bridgman (MVB) technique [1]. The measurements were made with the micro-focus beam line, housed in the Surrey Ion Beam centre, using $2 \mathrm{MeV}$ protons to excite the detector.

\section{EXPERIMENTAL METHOD}

Test detector structures were fabricated for this study using 6 $\mathrm{mm} \times 6 \mathrm{~mm} \times 2 \mathrm{~mm}$ pieces of $\mathrm{Cd}_{0.9} \mathrm{Zn}_{0.1}$ Te with Au contacts deposited on the (111) surfaces. The bulk resistivity of the material was measured from the device I-V characteristics and was found to be $1.3 \times 10^{10} \Omega \mathrm{cm}$. An initial characterisation of the charge transport properties of the material was carried out using an uncollimated ${ }^{241} \mathrm{Am}$ alpha particle source with an activity of $200 \mathrm{kBq}$. Alpha pulse height spectra were acquired using a shaping amplifier connected to a conventional multi-channel

Manuscript received July 17, 2008; revised September 08, 2008. Current version published December 31, 2008.

M. C. Veale, P. J. Sellin, J. Parkin, A. Lohstroh, and A. W. Davies are with the University of Surrey, Guildford, Surrey GU2 7XH, U.K. (e-mail: m.veale@surrey.ac.uk).

P. Seller is with STFC, Rutherford Appleton Laboratory, Chilton OX11 0QX, U.K.

Digital Object Identifier 10.1109/TNS.2008.2006745 analyzer; a shaping time of $6 \mu$ s was used which was sufficient enough to avoid any ballistic deficit at lower bias voltages. Hecht plots were produced from the pulse height data and were used to calculate electron and hole mobility-lifetime products $(\mu \tau)$. In addition alpha particle Time of Flight (TOF) measurements were performed using a waveform digitizer to simultaneously capture the preamplifier output pulses. By analyzing the measured pulse rise time as a function of applied voltage the electron and hole drift mobility $(\mu)$ were derived [2].

Spatially resolved charge transport data was obtained using high resolution IBIC mapping. These measurements were carried out at the nuclear microbeam facility at the University of Surrey [3]. A $2 \mathrm{MeV}$ proton beam was focused to a diameter of less than $5 \mu \mathrm{m}$ and used to scan an area of $\sim 2.5 \times 2.5 \mathrm{~mm}$ with a image resolution of $256 \times 256$ pixels. The beam current was significantly reduced so that single event pulses were recorded, with a proton rate incident on the sample of typically 500-1500 $\mathrm{Hz}$.

In the microbeam system the focused ion beam scans across the top contact of the device and the induced charge pulse for each proton interaction is measured using a charge sensitive preamplifier. The Surrey IBIC system operates simultaneously in both analogue and digital IBIC modalities. For analogue IBIC, the preamplifier pulse is passed through a spectroscopy amplifier which is connected to an MCA. This data produces high resolution maps of CCE as a function of bias voltage [4], [5].

The interaction depth of a $2 \mathrm{MeV}$ proton in $\mathrm{Cd}_{0.9} \mathrm{Zn}_{0.1}$ Te was calculated using SRIM [6], [7] and found to be $\sim 40 \mu \mathrm{m}$. Since this interaction depth is small compared to the sample thickness, the induced charge signal observed from cathode irradiation is essentially all due to electron transport, and conversely when the irradiated surface is made the anode the induced signal is due to hole transport.

By acquiring a sequence of CCE maps at different positive and negative polarity bias voltages, a pixel-by-pixel Hecht analysis was performed to produce maps of electron and hole $\mu \tau$. In this analysis the single charge carrier Hecht equation [8] is used

$$
\mathrm{CCE}=\frac{Q}{Q_{0}}=\frac{\mu_{e} \tau_{e} V}{d^{2}}\left[1-\exp \left(-\frac{d^{2}}{\mu_{e} \tau_{e} V}\right)\right]
$$

where $V$ is the applied voltage, and $d$ is the detector thickness. In the digital IBIC technique maps of the electron and hole drift time are constructed from the rise time of the digitized preamplifier pulses, analogous to a mapping TOF analysis, 


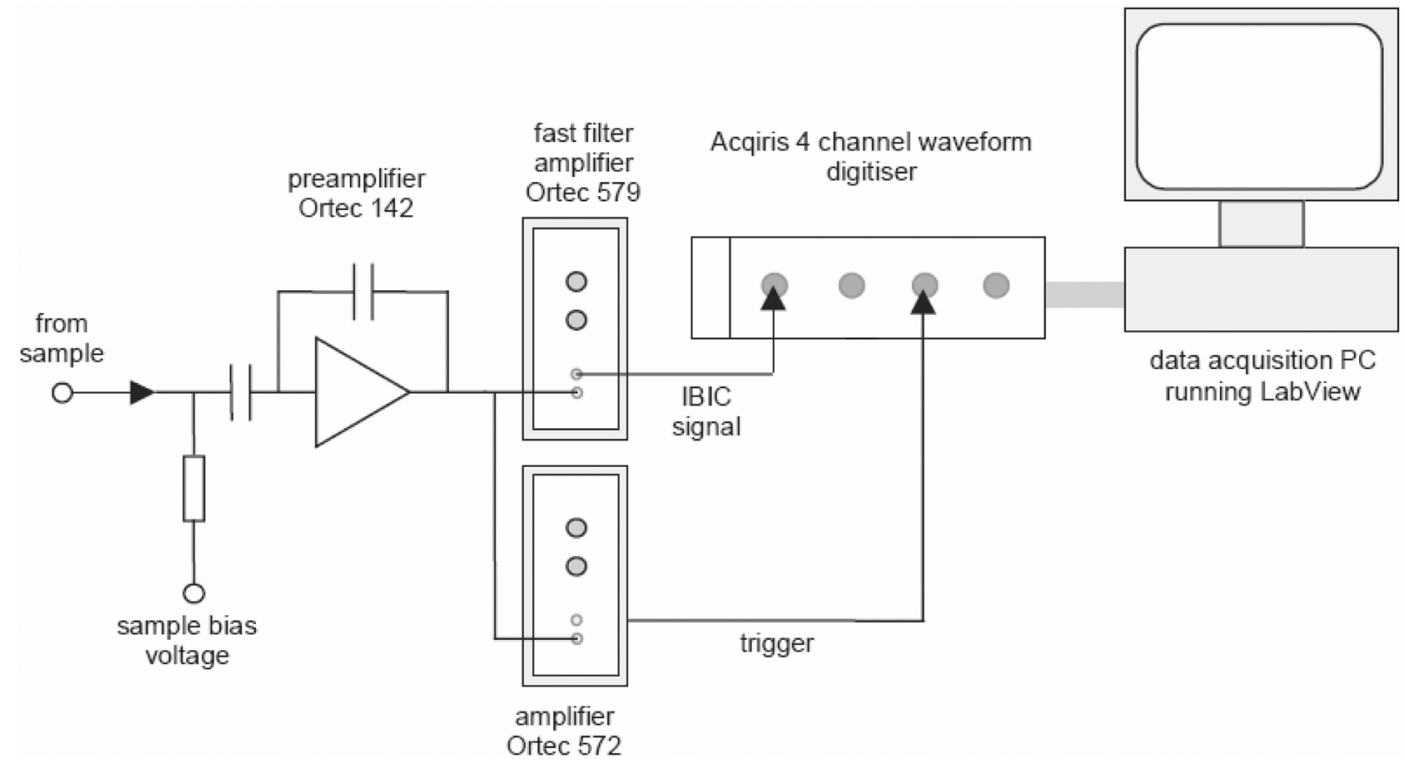

Fig. 1. Diagram of the Digital IBIC System [3].

where the drift mobility $\mu$ is related to the carrier drift time $\tau_{\mathrm{d}}$ by:

$$
\mu=\frac{d^{2}}{V \tau_{d}} .
$$

For each bias voltage a histogram of carrier drift time $\left(\tau_{\mathrm{d}}\right)$ is acquired for every pixel, and maps of either electron or hole drift mobility are calculated, which complement the $\mu \tau$ data. A schematic overview of the IBIC system is shown in Fig. 1.

\section{RESULTS AND DISCUSSION}

\section{A. Alpha Particle Spectroscopy}

Alpha particle pulse height spectra were acquired in vacuum, with the sample at room temperature, and the peak centroid was measured as a function of detector voltage. The peaks were calibrated into CCE using a pulser and a charge injector of known capacitance. Fig. 2 shows the resulting electron and hole CCE data acquired as a function of voltage. In each case the fit to the Hecht equation (1) is shown, giving values of $\mu \tau_{e}=1.2 \times 10^{-3}$ $\mathrm{cm}^{2} \mathrm{~V}^{-1}$ and $\mu \tau_{h}=5.8 \times 10^{-5} \mathrm{~cm}^{2} \mathrm{~V}^{-1}$, errors are calculated from the standard error in the regression. The values obtained are comparable with previously published values on similar CdZnTe material and demonstrate the large difference between the electron and hole transport [9], [10].

Alpha particle induced pulse shapes were captured simultaneously with the MCA data as a function of bias voltage. Carrier drift velocities were calculated from a Gaussian fit to histograms of pulse rise time. Using (2) the electron and hole drift mobility was measured from the linear regression to the data [2]. Fig. 3 shows the resulting fits, giving drift mobility values of $\mu_{e}=870$ and $\mu_{h}=16 \mathrm{~cm}^{2} \mathrm{~V}^{-1} \mathrm{~s}^{-1}$, the uncertainty in the values was calculated from the standard error in the linear regression. The $\mu \tau$ and drift mobility values both show that the material displays good electron transport properties but poor hole transport; which is similar to previous reported results [9], [10].

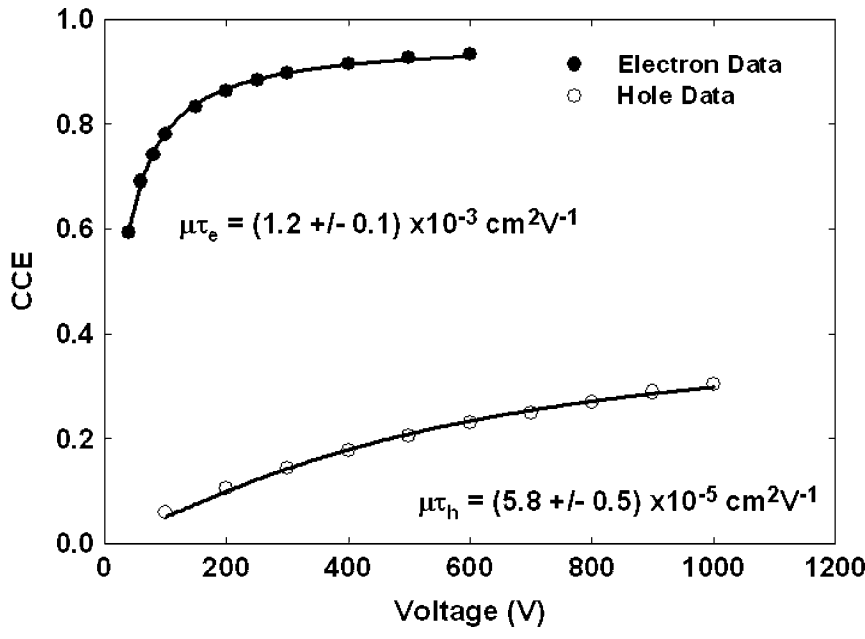

Fig. 2. Electron and Hole CCE-voltage data taken with $5.49 \mathrm{MeV} \alpha$ particles fitted with the single charge carrier Hecht Equation. The statistical uncertainty on each data point is negligible.

\section{B. IBIC Charge Transport Mapping}

1) Electron Data: IBIC imaging of a CdZnTe device was carried out using a microfocus $2 \mathrm{MeV}$ proton beam. The sample was irradiated with the beam direction orthogonal to the top contact, to which a bias voltage of $-800 \mathrm{~V}$ was applied. Fig. 4 shows the resulting electron CCE map from one corner of the device, covering an area of $2.5 \mathrm{~mm} \times 2.5 \mathrm{~mm}$. The edges of the metal electrode on the top surface are clearly visible in the image.

Overall the sample shows very uniform response, corresponding to a uniform electron drift length. Fig. 4 also shows the pulse height spectrum extracted from the indicated region (i) of the map, which shows a well resolved proton peak with a resolution of $20 \mathrm{keV}$ FWHM. Region (ii) at the bottom of the map is an area of no response due to the silver paint connection of the bond wire. 


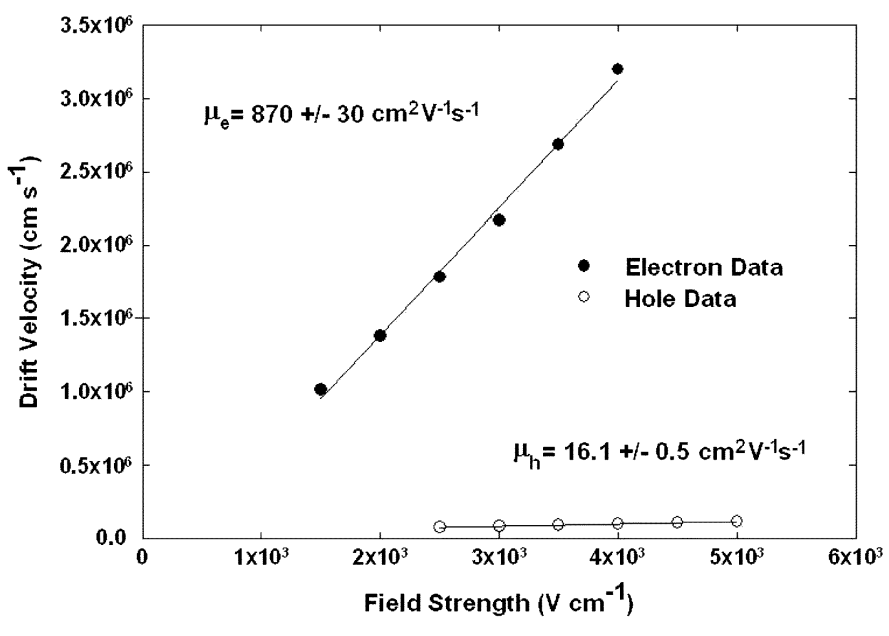

Fig. 3. Charge carrier drift velocity data taken from digital pulse analysis using $\alpha$ particles giving carrier drift mobility.
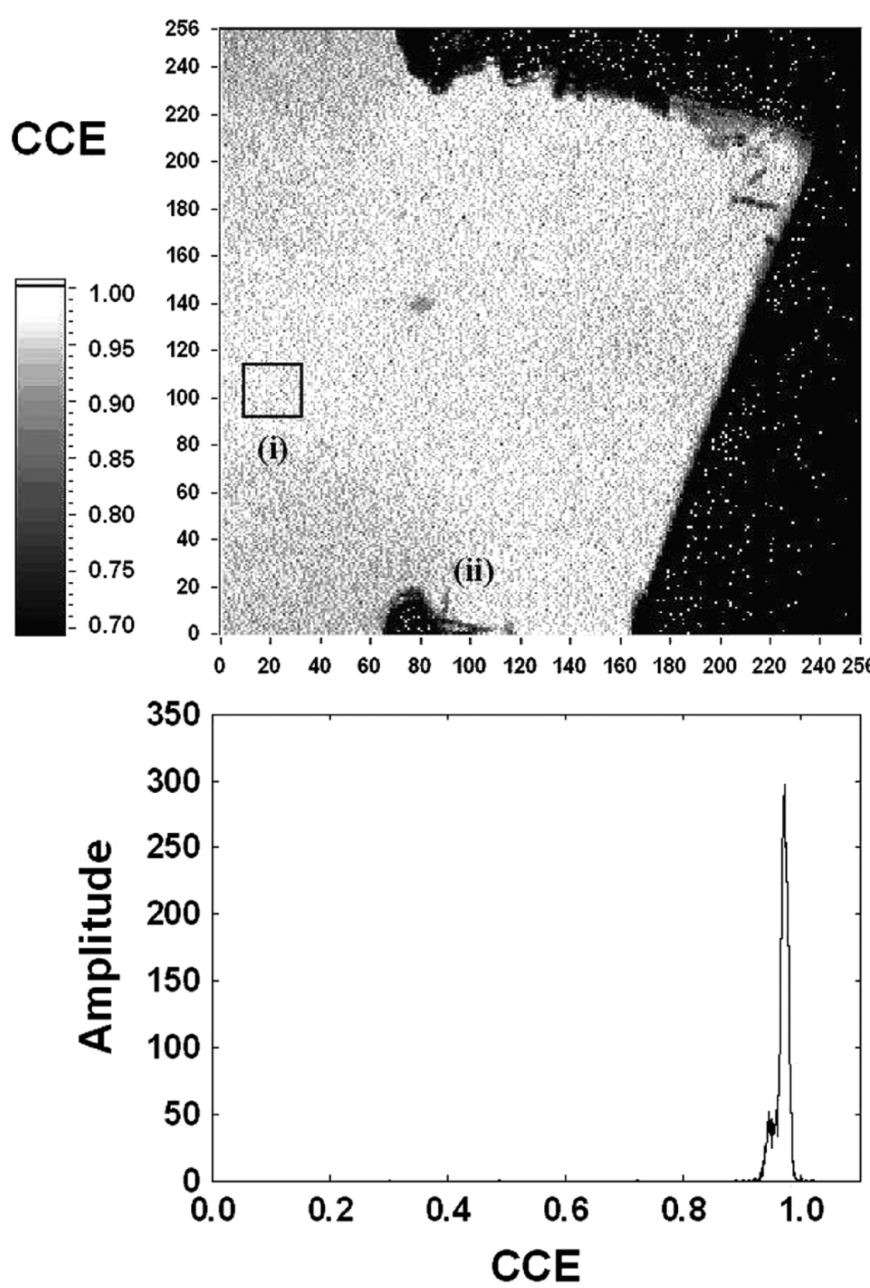

Fig. 4. (upper) IBIC map of electron CCE at $-800 \mathrm{~V}$; (lower) pulse height spectrum from the indicated region (i).

Fig. 5 shows a $\mu \tau_{\mathrm{e}}$ map which is constructed from six CCE maps taken at voltages between -30 and $-800 \mathrm{~V}$. Each pixel represents the $\mu \tau_{e}$ value calculated from the fit to (1). The map shows the uniformity of the electron charge transport, with an average $\mu \tau_{e}$ value across the imaged sample area of $0.9 \times 10^{-3}$
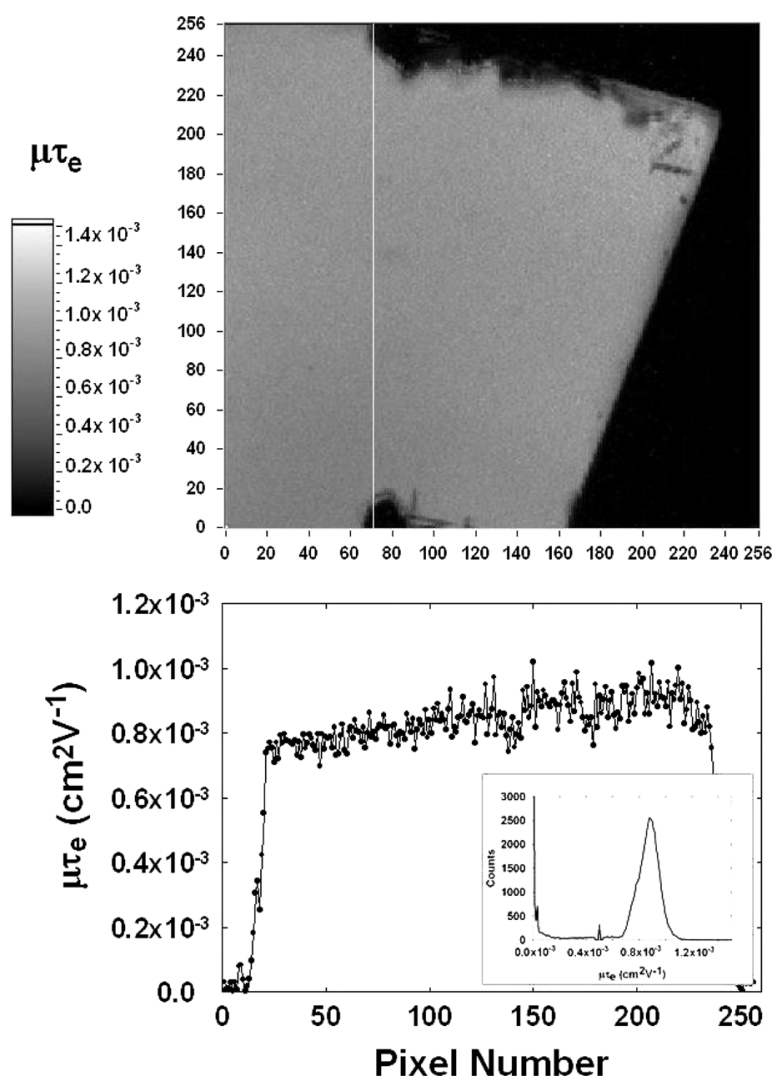

Fig. 5. (upper) Electron $\mu \tau$ map compiled from analogue IBIC CCE data sets; (lower) graph shows the cross-section along the white bar.

$\mathrm{cm}^{2} \mathrm{~V}^{-1}$, similar to the value obtained for the whole detector area from alpha particle measurements. Fig. 5 also shows a vertical cross-section through the map, showing a small variation in $\mu \tau_{e}$ value which increases slightly from the bottom to the top of the image.

A similar analysis of the digital IBIC data was carried out to produce a map of electron rise time at $-800 \mathrm{~V}$. Fig. 6 shows the resulting rise time map, which has a very uniform response with a mean electron pulse rise time of approximately $100 \mathrm{~ns}$. Also shown in Fig. 6 is a horizontal cross section through this map. indicating a slight reduction in drift time from 120 ns to 95 ns.

Despite this overall trend, the drift time response is uniform which confirms the homogeneous distribution of electron drift mobility in the sample. This is consistent with the observed uniformity in electron mobility-lifetime product obtained from the analogue IBIC data.

2) Hole Data: IBIC measurements were made from the same sample using an identical experimental setup but with a positive voltage applied to the top face of the sample. In this way the induced signals were due to hole drift through the material. Fig. 7 shows the hole CCE map taken at $+800 \mathrm{~V}$, acquired with the sample located in the same position as Fig. 4. In contrast to the electron data a large variation in the CCE is seen across the bulk of the detector, with regions of high and low response extending across distances of hundreds of micrometers. To illustrate this phenomena, Fig. 7 also shows pulse height spectra extracted from the two regions marked on the map as (1) and (2), which show mean CCE values of $42 \%$ and $65 \%$ respectively. 

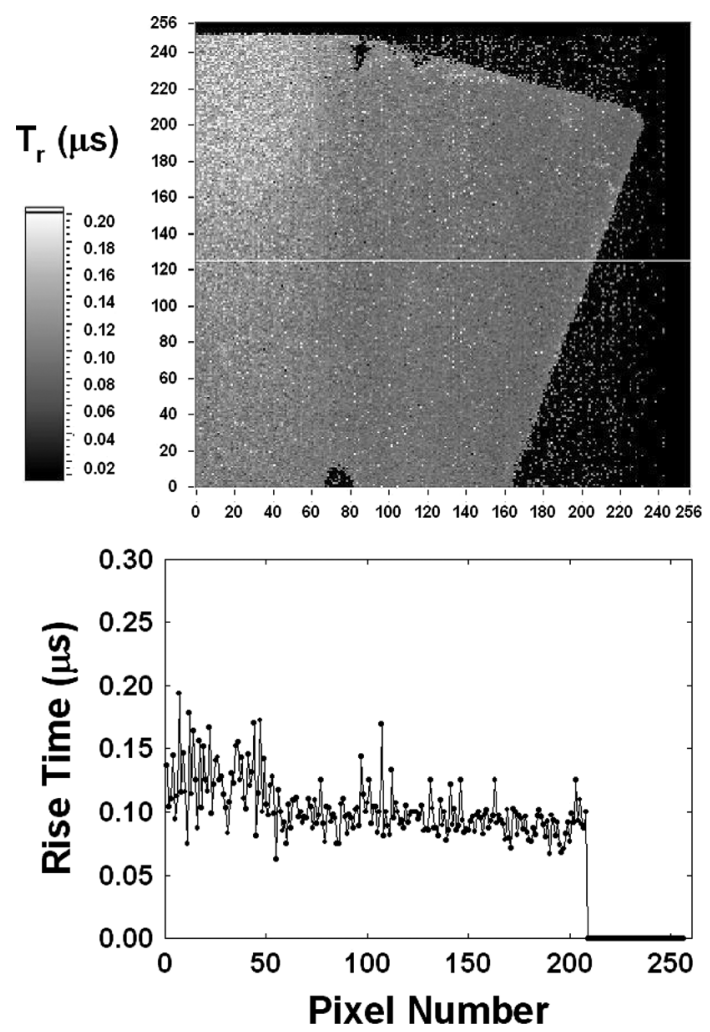

Fig. 6. (upper) Digital IBIC image showing electron pulse rise times at a bias of $-800 \mathrm{~V}$; (lower) cross-section along the white bar.
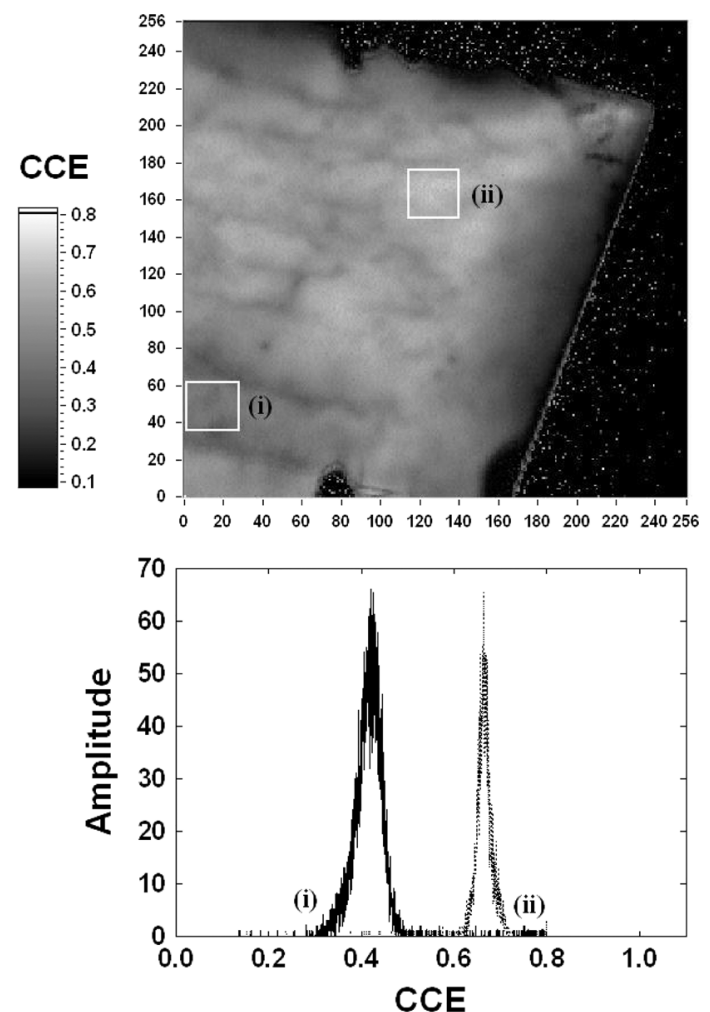

Fig. 7. (upper) Analogue IBIC image of hole CCE at $+800 \mathrm{~V}$; (lower) the spectral response of regions (i) and (ii).

The data demonstrates that the hole transport in this material shows a highly non-uniform response, with variations of up to $30 \%$ observed in hole drift length across the sample.
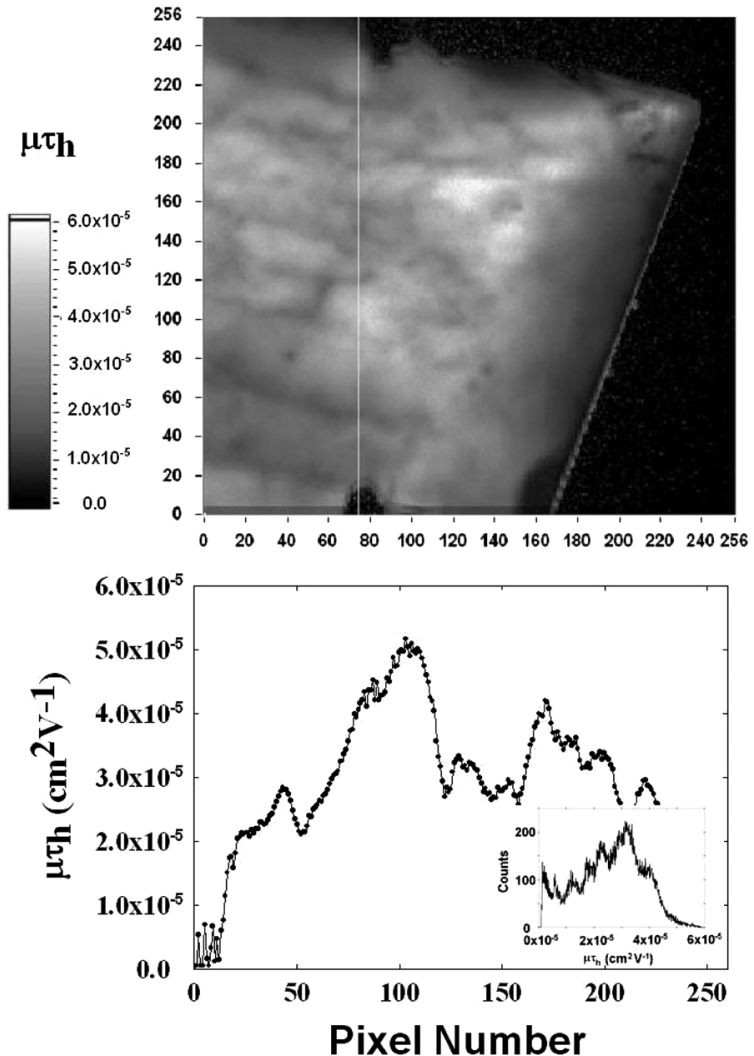

Fig. 8. (upper) Hole $\mu \tau$ map compiled from a number of CCE data sets; (lower) vertical cross-section along the white bar.

This is in contrast to the level of uniformity observed in the electron transport maps, and so cannot be due to lateral variations in electric field strength in the sample.

To confirm this effect, further hole CCE maps were acquired over a range of bias voltages from $+400 \mathrm{~V}$ to $+1000 \mathrm{~V}$ and a $\mu \tau_{\mathrm{h}}$ map was constructed by fitting each pixel value to (1). The resulting $\mu \tau_{\mathrm{h}}$ map is displayed in Fig. 8; which shows similar structural variations to those observed in Fig. 7. The typical range of $\mu \tau_{\mathrm{h}}$ values in the sample varies from $2 \times 10^{-5}$ $\mathrm{cm}^{2} \mathrm{~V}^{-1}$ to $5 \times 10^{-5} \mathrm{~cm}^{2} \mathrm{~V}^{-1}$. The spatial variation is shown in the vertical cross section, taken along the indicated line in Fig. 8 . The $2.5 \times$ variation between maximum and minimum hole mobility-lifetime product occurs over length scales which are far greater than that expected from extended defects such as inclusions in this material. Consequently these effects must be attributed to large scale variations of either drift mobility or carrier lifetime in the bulk material.

Further information is provided by studying the carrier drift time, as extracted from a map of hole rise time. Fig. 9 shows the rise time map produced for hole transport acquired simultaneously from the digital IBIC pulse shape data at $+800 \mathrm{~V}$.

The data shows a generally uniform response, in contrast to the $\mu \tau$ image, although two distinct region are visible: in region (i) the map shows uniform rise time response and this is reflected by the flat portion of the cross-section shown in Fig. 9. On the other hand region (ii) shows reduced hole rise times towards the edge of the sample. This is shown in the sharp fall-off in the cross-section at the edge of the sample, with hole drift 

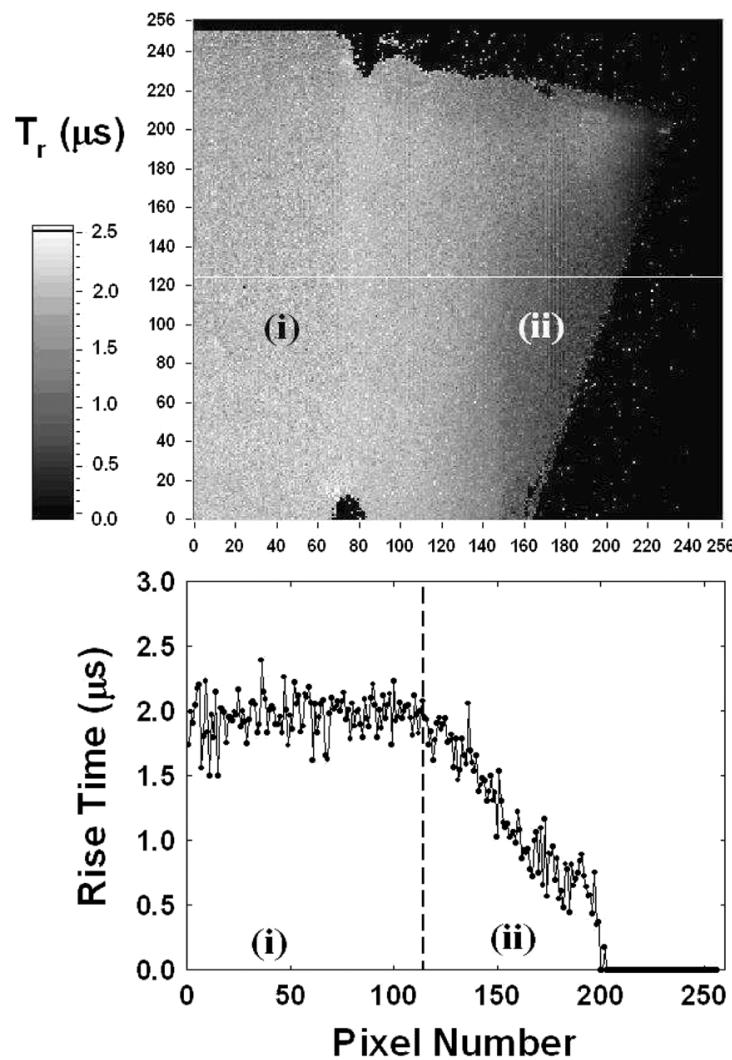

Fig. 9. (upper) Digital IBIC image of hole rise time at $+800 \mathrm{~V}$; (lower) the cross-section through the white bar. Region (i) shows an area of uniform response, (ii) shows a lifetime limited region.

times decreasing from $2 \mu$ s to $0.5 \mu \mathrm{s}$. Also, the individual pulse shapes from this region are uncharacteristically fast and with a low amplitude consistent with a significantly shorter hole lifetime in region (ii) of the device. Since this region of reduced hole lifetime follows the physical edge of the sample, it is most likely that this effect is due to saw damage or mechanical stress at the edge of the sample. However it is surprising that no similar effect is observed for the electron maps in this edge region.

Considering region (i) of the rise time map, the observed uniformity in hole drift time suggests that the hole drift mobility is uniform in this region. Therefore the observed variation in the hole mobility-lifetime product must be due to small fluctuations in the hole carrier lifetime [2]; this may indicate a spatial distribution of acceptor levels in the material.

Various candidate acceptor states have been reported which have a suitable capture cross section to influence hole carrier lifetimes [11], [12]. Spatial distributions of the transport properties of CdZnTe have also been demonstrated using focused X-rays [13], [14] and collimated alpha particles [15], [16]. These measurements have shown evidence for the localized effect of crystal defects, such as Tellurium Inclusions, and their associated trapping levels on the charge transport properties of CdZnTe.

\section{CONCLUSION}

We have presented charge transport maps using analogue and digital IBIC on a planar detector fabricated from vertical Bridgman CdZnTe. The room temperature electron mo- bility-lifetime product observed in this material is $1 \times 10^{-3}$ $\mathrm{cm}^{2} \mathrm{~V}^{-1}$ with a drift mobility of $870 \mathrm{~cm}^{2} \mathrm{~V}^{-1} \mathrm{~s}^{-1}$. This is an acceptable range of values for use in a gamma ray spectrometer with reasonable spectroscopic performance. However in contrast the hole transport in this material is poor, with a hole mobility-lifetime product of $5 \times 10^{-5} \mathrm{~cm}^{2} \mathrm{~V}^{-1}$ and a drift mobility of $17 \mathrm{~cm}^{2} \mathrm{~V}^{-1} \mathrm{~s}^{-1}$. IBIC imaging has provided useful information about the charge transport uniformity in this material, which shows a highly uniform electron response.

In contrast the hole transport has significant variations over length scales of hundreds of micrometers, these variations may be due to a spatial distribution of crystal defects and trapping levels.

\section{ACKNOWLEDGMENT}

The authors gratefully acknowledge supply of the CZT material from Yinnel Tech Inc. M. C. Veale acknowledges the Ph.D. studentship funded by STFC/Rutherford Appleton Laboratory.

\section{REFERENCES}

[1] L. Li et al., "Development of large single crystal (3-inch ingot) CdZnTe for large-volume nuclear radiation detectors," in Proc. SPIE, 2003, vol. 4784, pp. 76-83.

[2] A. Zuck, M. Schieber, O. Khakhan, and Z. Burshtein, "Polycrystalline HgI2 produced by physical vapour deposition," IEEE Trans. Nucl. Sci., vol. 50, no. 4, pp. 991-997, Aug. 2003.

[3] P. J. Sellin, A. Lohstroh, A. Simon, and M. B. H. Breese, "Digital IBIC-New spectroscopic modalities for ion-beam-induced charge imaging," Nucl. Instrum. Methods Phys. Res. A, vol. A521, pp. 600-607, 2004

[4] A. Lohstroh, P. J. Sellin, and A. Simon, "High-resolution mapping of the mobility-lifetime product in CdZnTe using a nuclear microprobe,' J. Phys. Condens. Matter, vol. 16, pp. S67-S73, 2004.

[5] M. B. Breese, D. N. Jamieson, and P. J. C. King, Materials Analysis Using a Nuclear Microprobe. New York: Wiley Interscience, 1996.

[6] J. P. Biersack and L. Haggmark, "A monte carlo computer program for the transport of energetic ions in amorphous targets," Nucl. Instrum. Methods, vol. 174, pp. 257-269, 1980.

[7] J. F. Ziegler, The Stopping and Range of Ions in Matter. New York: Pergamon, 1975-1985, vol. 2-6.

[8] K. Hecht, "Zum Mechanismus des lichtelektrischen Primärstroms in isolierenden Kristallen,” Z. Physik, vol. 77, pp. 235-245, 1932.

[9] L. Li et al., "New progress in large-size CZT $(\mathrm{Zn}=10 \%)$ single crystal growth using MVB technique for room temperature radiation detectors," in Proc. Nuclear Science Symp. Conf. Rec., Oct. 2004, vol. 4, pp. 2320-2323.

[10] B. W. Sturm, Z. He, T. H. Zurbuchen, and P. L. Koehn, "Investigation of the asymmetric characteristics and temperature effects of CdZnTe detectors," IEEE Trans. Nucl. Sci., vol. 52, no. 5, pp. 3074-3078, Oct. 2005.

[11] M. Fiederle, A. Fauler, J. Konrath, V. Babentsov, J. Franc, and R. B. James, "Comparison of undoped and doped high resistivity CdTe and (Cd, Zn)Te detector crystals," IEEE Trans. Nucl. Sci., vol. 51, no. 4, pp. 1864-1868, Aug. 2004.

[12] M. Ayoub et al., "Real defect concentration measurements of nuclear detector materials by the combination of PICTS and SCLC methods," Mater. Sci. Eng., vol. B83, pp. 173-179, Jan. 2001.

[13] G. A. Carini, A. E. Bolotnikov, G. S. Camarda, and R. B. James, "High-resolution X-ray mapping of CdZnTe detectors," Nucl. Instrum. Methods Phys. Res. A, vol. A579, pp. 120-124, Aug. 2007.

[14] G. A. Carini, A. E. Bolotnikov, G. S. Camarda, G. W. Wright, and R B. James, "Effect of Te precipitates on the performance of CdZnTe detectors," App. Phys. Lett., vol. 88, pp. 143515-, 2006.

[15] M. Amman, J. S. Lee, and P. N. Luke, "Electron trapping nonuniformity in high-pressure-Bridgman-grown CdZnTe," J. Appl. Phys., vol. 92, no. 3, pp. 3198-3206, Jun. 2002.

[16] S. A. Soldner, A. J. Narvett, D. E. Covalt, and C. Szeles, "Characterisation of the charge transport uniformity of $\mathrm{CdZnTe}$ crystals for large-volume nuclear detector applications," IEEE Trans. Nucl. Sci., vol. 51, no. 5, pp. 2443-2447, Oct. 2004. 